\title{
Влияние протонного облучения (15 МэВ) на низкочастотный шум мощных SiC MOSFETs
}

\author{
(C) А.А. Лебедев ${ }^{1}$, М.Е. Левинштейн ${ }^{1, \uparrow}$, П.А. Иванов ${ }^{1}$, В.В. Козловский ${ }^{2}$, А.М. Стрельчук ${ }^{1}$, \\ Е.И. Шабунина ${ }^{1}$, L. Fursin ${ }^{3}$ \\ ${ }^{1}$ Физико-технический институт им. А.Ф. Иоффре Российской академии наук, \\ 194021 Санкт-Петербург, Россия \\ ${ }^{2}$ Санкт-Петербургский политехнический университет Петра Великого, \\ 195251 Санкт-Петербург, Россия \\ ${ }^{3}$ United Silicon Carbide, Inc., Suite E Monmouth Junction, NJ 08852, USA \\ ฯ E-mail: melev@nimis.ioffe.ru
}

Поступила в Редакцию 23 июля 2019 г.

В окончательной редакции 29 июля 2019 г.

Принята к публикации 29 июля 2019 г.

\begin{abstract}
В диапазоне частот 1 Гц-50 кГц исследован низкочастотный шум в мощных $4 H$-SiC MOSFETs, подвергнутых облучению протонами с энергией 15 МэВ в диапазоне доз $\Phi 10^{12}-6 \cdot 10^{13} \mathrm{~cm}^{-2}$. Частотная зависимость спектральной плотности шума с хорошей точностью следует зависимости $S_{I} \propto 1 / f$. Прослежена корреляция между величиной тока насыщения выходной характеристики $I_{d}\left(V_{d}\right)$ и уровнем низкочастотного шума. В диапазоне доз $10^{12} \leq \Phi \leq 6 \cdot 10^{13} \mathrm{~cm}^{-2}$ значение тока насыщения изменяется в пределах $\sim 20 \%$, в то время как уровень шума меняется на 2 порядка. Из данных шумовой спектроскопии оценена плотность ловушек в подзатворном окисле $N_{t v}$. В необлученных структурах $N_{t v} \approx 5.4 \cdot 10^{18} \mathrm{~cm}^{-3} \cdot$ эВ $^{-1}$. При $\Phi=6 \cdot 10^{13} \mathrm{~cm}^{-2}$ величина $N_{t v}$ возрастает до значения $N_{t v} \approx 7.2 \cdot 10^{19} \mathrm{~cm}^{-3} \cdot{ }^{-1}$.
\end{abstract}

Ключевые слова: SiC MOSFETs, протонное облучение, низкочастотный шум.

DOI: 10.21883/FTP.2019.12.48610.9219

\section{1. Введение}

Мощные карбид-кремниевые ( $\mathrm{SiC})$ полевые транзисторы (MOSFETs) в настоящее время являются важнейшими компонентами силовой, высоковольтной и высокотемпературной электроники (см., например, [1]). При работе в условиях повышенной радиации (космическая электроника, ускорители частиц, ядерные электростанции) радиационная стойкость является одним из важнейших параметров, определяющих возможность применения того или иного полупроводникового прибора.

Исследованию стойкости SiC MOSFETs по отношению к различным типам радиационного излучения уделяется значительное внимание во всех промышленно развитых странах. Наибольшее внимание до настоящего времени уделялось исследованию радиационной стойкости $\mathrm{SiC}$ MOSFETs по отношению к облучению $\gamma$-частицами (см., например, [2-4]). Значительное число работ посвящено исследованию изменения параметров $\mathrm{SiC}$ MOSFETs под влиянием облучения быстрыми электронами [5-7].

Влияние облучения протонами на свойства $\mathrm{SiC}$ MOSFETs до последнего времени исследовалось только в низковольтных латеральных структурах с большой длиной затвора $L_{g} \approx 12-24$ мкм (см., например, $[8,9]$ ). Между тем дизайн современных мощных высоковольтных $\mathrm{SiC}$ MOSFETs принципиально отличен от дизайна латеральных структур. Прежде всего характерная длина затвора в современных $\mathrm{SiC}$ MOSFETs составляет $\sim 0.5$ мкм, что обусловливает возникновение прин- ципиально важных короткоканальных эффектов [10]. Кроме того, неотъемлемым элементом высоковольтных мощных MOSFETs является блокирующая приложенное напряжение слабо легированная дрейфовая область. Влияние облучения на параметры этой области могут оказаться чрезвычайно существенными [11].

Насколько нам известно, влияние протонного облучения на свойства современных высоковольтных мощных вертикальных $\mathrm{SiC}$ MOSFETs (VMOSFETs) изучалось только в работе [12]. В [12] было исследовано влияние облучения протонами (15 МэB) на крутизну, полевую подвижность носителей в канале, токи утечки и межэлектродную емкость. Показано, что мощные $\mathrm{SiC}$ VMOSFETs оказываются гораздо чувствительнее к облучению протонами, чем латеральные структуры. Доза $\Phi \approx 10^{14} \mathrm{~cm}^{-2}$ приводит к полной деградации мощного вертикального $4 H$-SiC MOSFETs, в то время как латеральные структуры сохраняют работоспособность при значительно бо́льших значениях Ф. Показано, что, как и в латеральных структурах, зависимость таких важнейших параметров, как ток насыщения и подвижность в канале, немонотонно зависят от $\Phi$ [9]. Качественно понятно, что наблюдающиеся зависимости обусловлены перезарядкой в результате облучения центров на границе окисел-канал и в самом окисле, а также, возможно, формированием новых заряженных центов $[9,13]$. Однако характер вновь образующихся при облучении центров и характеристики изменений зарядового состояния центров на границе окисел-канал до настоящего времени остаются неизученными. 
Измерения низкочастотного шума являются эффективным инструментом исследования плотности состояний на границе окисел-канал и плотности состояний в окисле затвора. Исследованию низкочастотного шума в Si MOSFETs и MOSFETs на основе соединений $\mathrm{A}^{\mathrm{III}} \mathrm{B}^{\mathrm{V}}$ посвящено очень много работ (см. [14-17] и ссылки в этих работах). Низкочастотный шум в $\mathrm{SiC}$ MOSFETs исследован значительно менее детально [18-20], и, насколько нам известно, какие-либо исследования низкочастотного шума в современных мощных высоковольтных SiC MOSFETs, облученных протонами, отсутствуют.

В настоящей работе впервые исследован низкочастотный шум в мощных высоковольтных (класс 1200 В) $4 H$-SiC VMOSFETs, подвергнутых облучению протонами с энергией 15 МэВ.

\section{2. Условия эксперимента}

Исследованные 4H-SiC MOSFETs класса 1200 В, изготовленные United Silicon Carbide, Inc. (USCi), представляют собой вертикальные структуры (VMOSFETs), состоящие из нескольких тысяч параллельно соединенных идентичных элементарных ячеек. Поперечное сечение одной из таких ячеек показано на рис. 1. Длина затвора $L_{g}$ составляет 0.5 мкм; полная ширина затвора структуры $W=490$ см. Толщина слоя окисла $d=60$ нм. Концентрация акцепторов в карманах $p$-типа, $N_{A}=2 \cdot 10^{17} \mathrm{~cm}^{-3}$. Толщина $H_{d}$ слабо легированного дрейфового слоя с уровнем легирования $N_{d}=8 \cdot 10^{15} \mathrm{~cm}^{-3}$ составляет 9 мкм. Длина дрейфового слоя $L_{d}=1$ мкм.

Приборы облучались протонами на портативном циклотроне MGTs-20 в импульсном режиме. Энергия протонов составляла 15 МэВ. Диаметр пучка составлял 30 мм, плотность тока в протонном пучке лежала в диапазоне $10-100 \mathrm{HA} / \mathrm{cm}^{2}$. Длительность импульсов составляла 2 мс, частота повторения - 100 Гц. Дозы облучения $\Phi$ варьировались в диапазоне $10^{12}-10^{14} \mathrm{~cm}^{-2}$. Облучение проводилось в режиме форсированного охла-

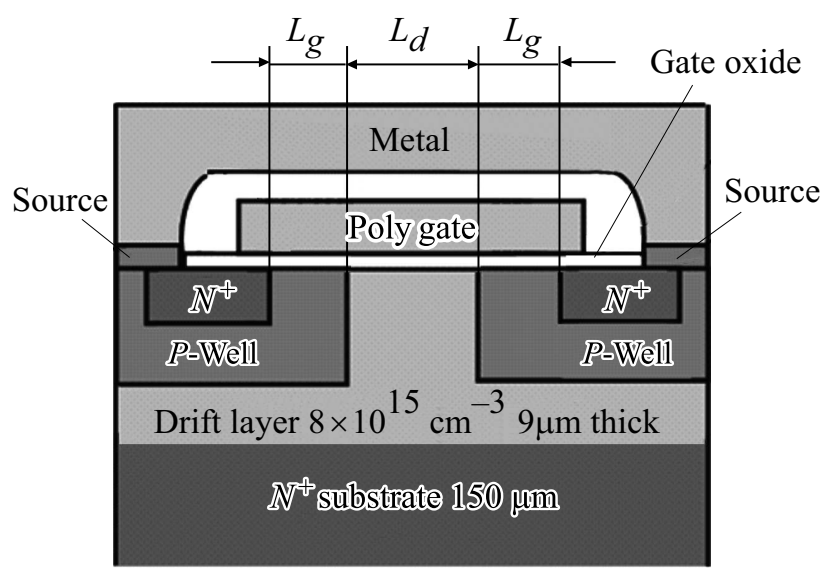

Рис. 1. Схематическое изображение элементарной ячейки исследованных $4 H$-SiC VMOSFETs. ждения мишени; температура образцов в процессе облучения не превышала $30^{\circ} \mathrm{C}$. Длина пробега протонов, рассчитанная с помощью коммерческой программы SRIM [21], составляла 1 мм. Таким образом, дефекты вводились в материал структуры однородно.

Низкочастотный шум измерялся в схеме с заземленным истоком в диапазоне частот 1 Гц-10кГц при комнатной температуре. Флуктуации напряжения измерялись на высокостабильном нагрузочном сопротивлении $R_{L}$, включенном последовательно со стоковым электродом прибора. Спектральная плотность флуктуаций тока рассчитывалась как $S_{I}=S_{V}\left[\left(R_{L}+R_{d}\right) /\left(R_{L} R_{d}\right)\right]^{2}$, где $R_{d}$ - дифференциальное сопротивление исток-сток.

\section{3. Результаты и обсуждение}

На рис. 2 приведены выходные характеристики исследуемых VMOSFETs при напряжении на затворе $V_{g}=5 \mathrm{~B}$ для нескольких дозах облучения Ф. Из рисунка ясно видно, что зависимость выходного тока $I_{d}$ от дозы $\Phi$ не монотонна. Минимальная доза $\Phi=10^{12} \mathrm{~cm}^{-2}$ приводит к уменьшению выходного тока. При увеличении $\Phi$ до $5 \cdot 10^{12} \mathrm{~cm}^{-2}$ выходной ток возрастает до значений, превышающих значения $I_{d}$ при $\Phi=0$. С дальнейшим ростом $\Phi$ выходной ток монотонно уменьшается. Немонотонная зависимость $I_{d}(\Phi)$ наблюдалась ранее как в латеральных, так и в мощных вертикальных $4 H-\mathrm{SiC}$ MOSFETs $[9,12]$.

Заметим, что, как показано в работе [12], дозе $\Phi=10^{14} \mathrm{~cm}^{-2}$ соответствует полная деградация прибора: сопротивление дрейфового слоя возрастает до значений $\sim 10^{6} \mathrm{OM}$ за счет практически полного захвата свободных электронов на создаваемые протонным облучением акцепторные уровни $Z_{1 / 2}$ [22,23]. Дозе $\Phi=6 \cdot 10^{13} \mathrm{~cm}^{-2}$ соответствует заметная деградация крутизны и полевой подвижности прибора [12].

На вставке к рис. 2 показана переходная характеристика прибора в области малых значений тока стока $I_{d}$ (квазилинейная часть выходной характеристики). Интерполяция зависимости $I_{d}\left(V_{g}\right)$ к значению $V_{g}=0$ определяет пороговое напряжение $V_{\text {th }}[12,13]$. Видно, что величина $V_{\text {th }}$ оказывается практически одинаковой для всех значений $I_{d}$ в области квазилинейности. Значение $V_{\text {th }}$ слабо зависит от дозы $\Phi$ и в области актуальных значений $Ф$ лежит в пределах $1.8-2$ В.

Из рис. 2 видно, что выходные характеристики прибора демонстрируют неполное насыщение тока стока даже при достаточно больших напряжениях на стоке $V_{d}$. Кроме того, даже при относительно малых значениях $V_{d}$ зависимость $I_{d}\left(V_{d}\right)$ нелинейна (сублинейна). Такие выходные характеристики свидетельствуют о значительной плотности ловушек на интерфейсе канал-окисел и(или) в самом окисле затвора [24].

На рис. 3 представлены частотные зависимости спектральной плотности токового шума для двух значений напряжения на затворе $V_{g}$, соответствующих до- 


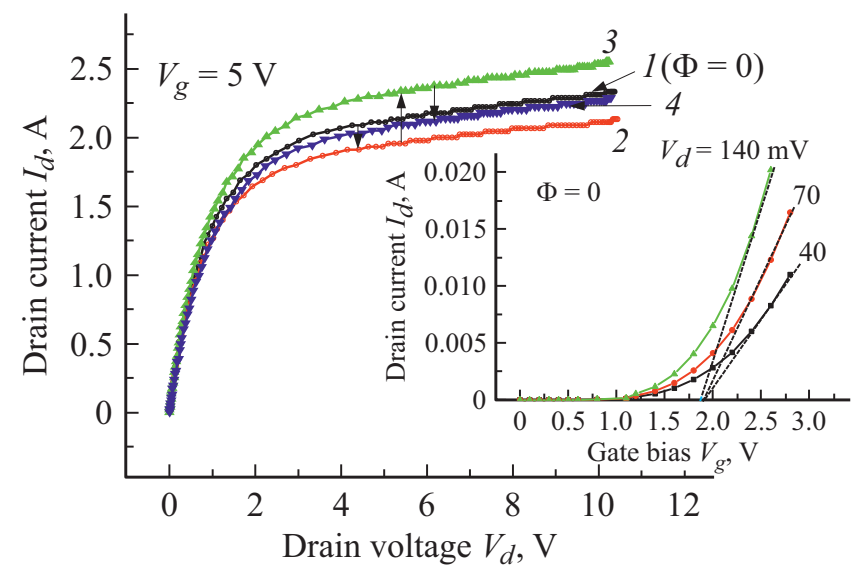

Рис. 2. Выходные характеристики исследуемых VMOSFETs при напряжении на затворе $V_{g}=5 \mathrm{~B}$ для нескольких доз облучения $\Phi\left(\mathrm{cm}^{-2}\right): 1-0,2-10^{12}, 3-5 \cdot 10^{12}, 4-6 \cdot 10^{13}$. Стрелки наглядно иллюстрируют немонотонное изменение выходного тока с изменением дозы $Ф$. На вставке приведены переходные характеристики прибора $I_{d}\left(V_{g}\right)$ при $\Phi=0$ для различных значений напряжения на стоке $V_{d}(\mathrm{MB}): 1-40$, $2-70,3-140$.

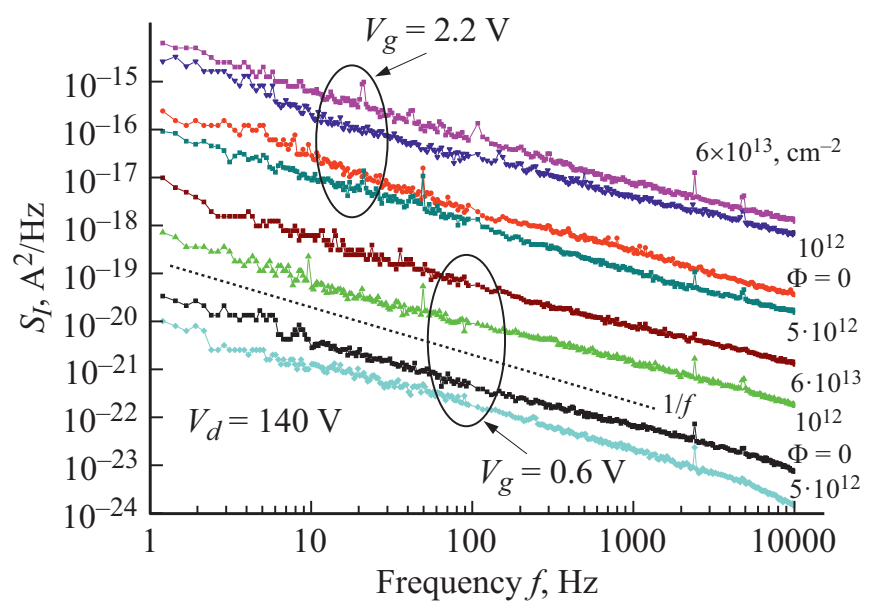

Pис. 3. Частотные зависимости спектральной плотности токового шума $S_{I}$ в допороговой области $\left(V_{g}=0.6 \mathrm{~B}<V_{\mathrm{th}}\right)$ и при $V_{g}=2.2 \mathrm{~B}>V_{\text {th }}$ для четырех значений дозы $\Phi$. Для всех кривых напряжение на стоке $V_{d}=140 \mathrm{MB}$. Пунктирная линия соответствует наклону $S_{I} \propto 1 / f$.

пороговому режиму $\left(V_{g}=0.6 \mathrm{~B}<V_{\text {th }}\right)$ и значению $V_{g}$, превышающему пороговое значение $\left(V_{g}=2.2 \mathrm{~B}>V_{\text {th }}\right)$. Представленные зависимости измерены при напряжении на стоке $V_{d}=140 \mathrm{MB}$ для четырех значений дозы $\Phi$. Видно, что и в допороговой области, и при напряжениях на затворе, превышающих пороговое значение $V_{\text {th }}$, зависимости $S_{I}(f)$ с хорошей точностью следуют закону $S_{I} \propto 1 / f$ для частот анализа $f \leq 10^{4} \Gamma$ ц.

Сравнивая данные, приведенные на рис. 2 и 3 , легко убедиться, что существует корреляция между величиной тока насыщения и уровнем низкочастотного шума. После облучения дозой $\Phi=10^{12} \mathrm{~cm}^{-2}$ ток на- сыщения падает, а уровень шума возрастает. Облучение дозой $\Phi=5 \cdot 10^{12} \mathrm{~cm}^{-2}$ приводит к возрастанию тока насыщения; при этом уровень шума заметно падает. С дальнейшим ростом дозы $\Phi$ шум монотонно возрастает. Следует заметить, что, как и следовало ожидать, низкочастотный шум оказывается заметно более чувствительным параметром, чем ток насыщения в области доз $\Phi$, где сохраняется работоспособность прибора: $0 \leq \Phi \leq 4 \cdot 10^{13} \mathrm{~cm}^{-2}$ [12]. Так, в диапазоне доз $0 \leq \Phi \leq 6 \cdot 10^{13} \mathrm{~cm}^{-2}$ значение тока насыщения изменяется в $\sim 1.2$ раза (рис. 2 ), в то время как уровень шума меняется на 2 порядка (рис. 3).

На рис. 4 представлена зависимость относительной спектральной плотности шума от тока стока $I_{d}$ при постоянном напряжении на стоке $V_{d}=140 \mathrm{MB}$ (ток стока меняется за счет изменения напряжения на затворе $V_{g}$ ). Частота анализа $f=1.22$ ц ; $V_{d}=140 \mathrm{MB}$.

Для современных кремниевых MOSFETs, характеризующихся относительно малой плотностью локализованных состояний на границе канал-окисел и небольшой плотностью ловушек в окисле затвора $N_{t v}$, зависимость $S_{I} / I_{d}^{2}\left(I_{d}\right)$, как правило, следует закону $S_{I} / I_{d}^{2} \propto 1 / I_{d}^{2}$. Как уже отмечалось ранее, для $\mathrm{SiC}$ MOSFETs, так же как для аморфных и поликристаллических тонкопленочных транзисторов, характерны зависимости $S_{I} / I_{d}^{2}\left(I_{d}\right) \propto 1 / f_{d}^{0.5}[18,25,26]$, что связано с сильной зависимостью плотности локализованных состояний от энергетического зазора между уровнем Ферми и дном зоны проводимости. Как видно из рис. 4, для исследуемых структур зависимость $S_{I} / I_{d}^{2}\left(I_{d}\right) \propto 1 / f_{d}^{0.5}$ характерна для всех значений доз от $\Phi=0$ до $\Phi=6 \cdot 10^{13} \mathrm{~cm}^{-2}$.

Используя данные, представленные на рис. 4, можно оценить эффективную плотность ловушек в подзатворном окисле $N_{t v}$. В соответствии с моделью [27], в режиме сильной инверсии $\left(V_{g}-V_{\text {th }} \gg k T\right)$ между плотностью ловушек в окисле и относительной спектральной плотностью шума $S_{I} / I^{2}$ выполняется соотношение

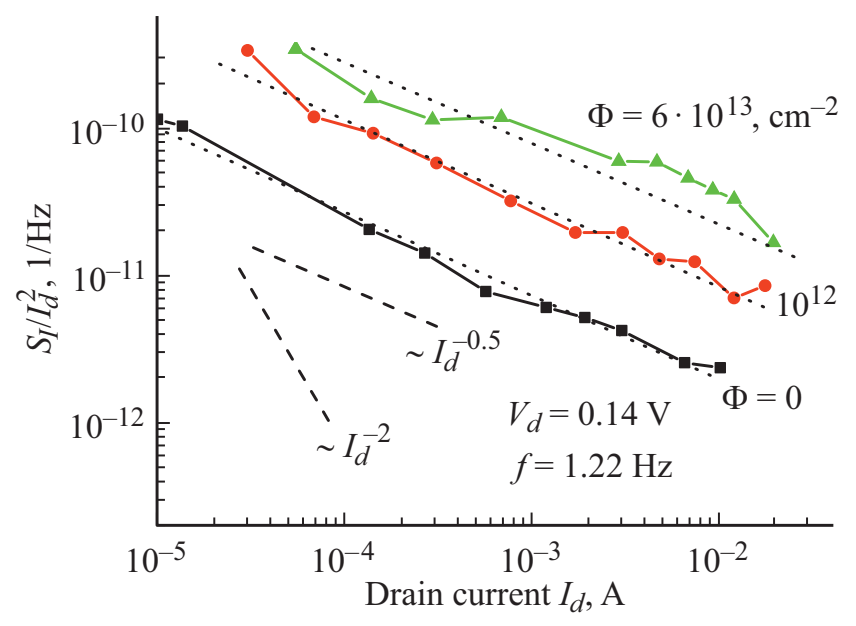

Рис. 4. Зависимость относительной спектральной плотности шума $S_{I} / I_{d}^{2}$ от тока стока. 
(см., например, [28])

$$
\frac{S_{I}}{I_{d}^{2}} \approx \frac{\lambda k T N_{t v}}{f W L_{g} n_{s}^{2}} \approx \frac{\lambda k T e^{2} d^{2} N_{t v}}{f W L_{g}\left(\varepsilon_{0} \varepsilon\right)^{2}\left(V_{g}-V_{\mathrm{th}}\right)^{2}},
$$

где $e-$ элементарный заряд, $\lambda \approx 10^{-8}-$ постоянная туннелирования, $\varepsilon_{0}$ - диэлектрическая постоянная вакуума, $\varepsilon=3.8-$ диэлектрическая постоянная окисла,

$$
n_{s}=\frac{\varepsilon_{o x} \varepsilon_{0}\left(V_{g}-V_{\mathrm{th}}\right)}{e d},
$$

$n_{s}$ - поверхностная плотность электронов в канале.

Для исследованных структур, как в допороговой области, так и при значениях $V_{g}$, превышающих пороговое значение $V_{\mathrm{th}}$, спектральная плотность шума $S_{I}$ при постоянном значении $V_{g}$ с хорошей точностью следует зависимости $S_{I} \propto I_{d}^{2}[18]$.

При $\quad \Phi=0, \quad V_{g}=2.2 \mathrm{~B}, \quad\left(V_{g}-V_{\text {th }} \approx 0.4 \mathrm{~B}\right) \quad$ и $f=1.22$ Гц, $S_{I} / I^{2} \approx 2.3 \cdot 10^{-12} 1 / \Gamma_{ц} \quad$ (рис. 4$), \quad$ и $N_{t v} \approx 5.3 \cdot 10^{24} \mathrm{M}^{-3} \cdot$ эB $^{-1} \approx 5.4 \cdot 10^{18} \mathrm{M}^{-3} \cdot{ }^{-1} \mathrm{~B}^{-1}$. Это значение с разумной точностью согласуется с величиной $N_{t v} \approx 10^{19} \mathrm{M}^{-3} \cdot$ эB $^{-1}, \quad$ полученной для латеральных $\mathrm{SiC}$ MOSFETs в работе [19]. При $\Phi=6 \cdot 10^{13} \mathrm{~cm}^{-2}$ оценка для плотности ловушек дает значение $N_{t v} \approx 7.2 \cdot 10^{19} \mathrm{M}^{-3} \cdot{ } \mathrm{B}^{-1}$.

\section{4. Заключение}

Впервые исследован низкочастотный шум в мощных 4H-SiC MOSFETs, подвергнутых облучению протонами (с энергией $15 \mathrm{MэB}$ ) в диапазоне доз $10^{12} \leq \Phi \leq 6 \cdot 10^{13} \mathrm{~cm}^{-2}$. Максимальное значение дозы $\Phi$ близко в предельному значению $\Phi \approx 10^{14} \mathrm{~cm}^{-2}$, соответствующему полной деградации прибора. Во всем исследованном диапазоне частот анализа, напряжений на затворе и смещений сток-исток частотная зависимость спектральной плотности шума с хорошей точностью следует зависимости $S_{I} \propto 1 / f$. Прослежена корреляция между величиной тока насыщения выходной характеристик прибора и уровнем низкочастотного шума. После облучения минимальной дозой $\Phi=10^{12} \mathrm{~cm}^{-2}$ ток насыщения падает, а уровень шума возрастает. Облучение дозой $\Phi=5 \cdot 10^{12} \mathrm{~cm}^{-2}$ приводит к возрастанию тока насыщения; при этом уровень шума заметно падает. С дальнейшим ростом дозы $\Phi$ шум монотонно возрастает. В диапазоне доз $10^{12} \leq \Phi \leq 6 \cdot 10^{13} \mathrm{~cm}^{-2}$ значение тока насыщения изменяется в пределах $\sim 20 \%$, в то время как уровень шума меняется на 2 порядка. Из данных шумовой спектроскопии оценена плотность ловушек в подзатворном окисле $N_{t v}$. В необлученных структурах $N_{t v} \approx 5.4 \cdot 10^{18} \mathrm{M}^{-3} \cdot$ эВ ${ }^{-1}$. При $\Phi=6 \cdot 10^{13} \mathrm{~cm}^{-2}$ величина возрастает до значения $N_{t v} \approx 7.2 \cdot 10^{19} \mathrm{M}^{-3} \cdot{ }^{-} \mathrm{B}^{-1}$.

\section{Финансирование работы}

Работа частично поддержана грантом Российского научного фонда, проект № 16-12-10106.

\section{Конфликт интересов}

Авторы заявляют, что у них нет конфликта интересов.

\section{Список литературы}

[1] T. Ohshima, T. Yokoseki, K. Murata, T. Matsuda, S. Mitomo et al. Jpn. J. Appl. Phys., 55, 01AD01 (2016).

[2] M. Masunaga, S. Sato, R. Kuwana, I. Hara, A. Shima. Mater. Sci. Forum, 924, 984 (2018).

[3] K. Murata et al. Phys. Status Solidi A, 214, 1600446 (2017).

[4] A. Takeyama et al. Jpn. J. Appl. Phys., 55, 104101 (2016).

[5] V.V. Kozlovski, A.A. Lebedev, E.V. Bogdanova. J. Appl. Phys., 117, 155702 (2015).

[6] S. Popelka, P. Hazdra. Mater. Sci. Forum, 858, 856 (2016).

[7] В.В. Козловский, А.А. Лебедев, В.Н. Ломасов, Е.В. Богданова, Н.В. Середова. ФТП, 48, 1033 (2014).

[8] M. Florentin, M. Alexandru, A. Constant, B. Schmidt, P. Godignon. Mater. Sci. Forum, 806, 121 (2015).

[9] M. Alexandru, M. Florentin, A. Constant, B. Schmidt, P. Michel, P. Godignon. IEEE Trans. Nucl. Sci., 61, 1732 (2014).

[10] P. Valizadeh. Field Effect Transistors, A Comprehensive Overview: From Basic Concepts to Novel Technologies (N.Y., Wiley, 2016).

[11] A.A. Lebedev, A.I. Veinger, D.V. Davydov, V.V. Kozlovski, N.S. Savkina, A.M. Strel'chuk. J. Appl. Phys., 88, 6285 (2000).

[12] A.A. Lebedev, V.V. Kozlovski, M.E. Levinshtein, P.A. Ivanov, A.M. Strel'chuk, A.V. Zubov, L. Fursin. Semicond. Sci. Technol., 34, 045004 (2019).

[13] S.L. Rumyantsev, M.S. Shur, M.E. Levinshtein, P.A. Ivanov, J.W. Palmour, A.K. Agarwal, B.A. Hull, Sei-Hyung Ryu. Semicond. Sci. Technol., 24, 075011 (2009).

[14] Z. Celik-Butler. IEE Proc. Circuits, Dev. Syst., 149, 23 (2002).

[15] M. Haartman, M. Östling. Low-Frequency Noise in Advanced MOS Devices. In: Analog Circuits and Signal Processing (Springer, 2007).

[16] Si Mengwei, N.J. Conrad, S. Sanghoon, Gu Jiangjiang, Zhang Jingyun, M.A. Alam, P.D. Ye. IEEE Trans. Electron Dev., 62, 3508 (2015).

[17] V. Passi, J.P. Raskin. Semicond. Sci. Technol., 32, 123004 (2017).

[18] S.L. Rumyantsev, M.S. Shur, M.E. Levinshtein, P.A. Ivanov, J.W. Palmour, M.K. Das, B.A. Hull. J. Appl. Phys., 104, 094505 (2008).

[19] S.L. Rumyantsev, M.S. Shur, M.E. Levinshtein, P.A. Ivanov, J.W. Palmour, A.K. Agarwal, S. Dhar. Semicond. Sci. Technol., 26, 085015 (2011).

[20] X.Zh. Cher, Sh. Xiao, X.Zh. En, D.M. Fleetwood, R.D. Schrimpf, S.A. Francis, T. Roy, S. Dhar, Ryu Sei-Hyung, S.T. Pantelides. IEEE Trans. Electron Dev., 60, 2361 (2013).

[21] J.F. Ziegler, J.P. Biersack, U. Littmark. The Stopping and Range of Ions in Matter (N.Y., Pergamon Press, 1985).

[22] A. Castaldini, A. Cavallini, L. Rigutti, F. Nava. Appl. Phys. Lett., 85, 3780 (2004).

[23] H. Kaneko, T. Kimoto. Appl. Phys. Lett., 98, 262106 (2011).

[24] M. Shur. Physics of semiconductor devices (Prentice Hall, Englewood Cliffs, New Jersey, 1990).

[25] M. Rahal, M. Lee, A.P. Burdett. IEEE Trans. Electron Dev., 49, 319 (2002). 
[26] D. Rigaud, M. Valenza, J. Rhayem. IEE Proc. - Circuits, Dev. Syst., 149, 75 (2002).

[27] A.L. McWhorter. Proc. Conf. on the Phys. Semicond. Surfaces (Philadelphia, 1956).

[28] R. Jayaraman, C.G. Sodini. IEEE Trans. Electron Dev., 36, 1773 (1989).

Редактор Г.А. Оганесян

\title{
Effect of proton (15 MeV) irradiation on low frequency noise in power SiC MOSFETs
}

\author{
A.A. Lebedev' ${ }^{1}$, M.E. Levinshtein'1 P.A. Ivanov', \\ V.V. Kozlovski ${ }^{2}$, A.M. Strel'chuk', \\ E.I. Shabunina ${ }^{1}$, L. Fursin ${ }^{3}$ \\ ${ }^{1}$ loffe Institute, \\ 194021 St. Petersburg, Russia \\ 2 Peter the Creat St. Petersburg Polytechnic University, \\ 194021 St. Petersburg, Russia \\ ${ }^{3}$ United Silicon Carbide, Inc., Suite E Monmouth \\ Junction, NJ 08852, USA
}

Abstract Low frequency noise has been studied in power $4 H$-SiC MOSFETs after proton $(15 \mathrm{MeV})$ irradiation. The noise was studied at room temperature in the frequency range $1 \mathrm{~Hz}-50 \mathrm{kHz}$ after irradiation with doses of $10^{12} \leq \Phi \leq 6 \cdot 10^{13} \mathrm{~cm}^{-2}$. Frequency dependence of the spectral noise density $S_{I}$ follows with good accuracy to the law $S_{I} \propto 1 / f$. The correlation between the saturation current of the output characteristics of $I_{d}\left(V_{d}\right)$ and the level of low-frequency noise is established. In the dose range $\Phi$ studied the value of the saturation current varies within about $20 \%$, while the noise level changes by 2 orders of magnitude. From the data of noise spectroscopy, the density of traps in the gate oxide $N_{t v}$ was estimated. In non-irradiated structures $N_{t v} \approx 5.4 \cdot 10^{18} \mathrm{~cm}^{-3} \cdot \mathrm{eV}^{-1}$, at $\Phi=6 \cdot 10^{13} \mathrm{~cm}^{-2}, N_{t v}$ increases to a value of $N_{t v} \approx 7.2 \cdot 10^{19} \mathrm{~cm}^{-3} \cdot \mathrm{eV}^{-1}$. 